\title{
Positionspapier zur Bedeutung der forcierten Vitalkapazität für Patienten mit idiopathischer Lungenfibrose (IPF)
}

\author{
Position Paper: Significance of the Forced Vital Capacity in Idiopathic \\ Pulmonary Fibrosis
}

Autoren

Institute
J. Behr ${ }^{1}$, F. Bonella ${ }^{2}$, R. Bonnet ${ }^{3}$, S. Gläser ${ }^{4}$, C. Grohé ${ }^{5}$, A. Günther ${ }^{6}$, D. Koschel ${ }^{7}$, M. Kreuter ${ }^{8}$, D. Kirsten ${ }^{9}$, C. Krögel ${ }^{10}$, P. Markartt ${ }^{11}$, J. Müller-Quernheim ${ }^{12}$, C. Neurohr ${ }^{13}$, M. Pfeifer ${ }^{14}$, A. Prasse ${ }^{15}$, N. Schönfeld ${ }^{16}$, J. Schreiber ${ }^{17}$, H. Wirtz ${ }^{18}$, C. Witt ${ }^{19}$, U. Costabel ${ }^{20}$

Die Institutsangaben sind am Ende des Beitrags gelistet.
Bibliografie

DOI http://dx.doi.org/

10.1055/s-0034-1392602

Online-Publikation: 30.7.2015

Pneumologie 2015; 69: 455-458

(c) Georg Thieme Verlag KG

Stuttgart · New York

ISSN 0934-8387

\section{Korrespondenzadresse}

Prof. Dr. med. Jürgen Behr

Klinikum der Universität

München

Medizinischen Klinik und

Poliklinik V

Marchioninistr. 15

81377 München

Juergen.Behr@med.uni-

muenchen.de

\section{Zusammenfassung \\ $\nabla$}

Die Spirometrie ist ein hervorragend standardisiertes Messverfahren, mit dem die forcierte Vitalkapazität (FVC) mit hoher Präzision und Reproduzierbarkeit bestimmt werden kann. Speziell bei Patienten mit idiopathischer pulmonaler Fibrose (IPF) reflektiert die FVC und deren Abfall über die Zeit den zugrunde liegenden Krankheitsprozess, der durch Vernarbung der Alveolen und Schrumpfung der Lungen charakterisiert ist. Dementsprechend zeigen zahlreiche Studien einen konsistenten Zusammenhang zwischen der Abnahme der FVC im Zeitverlauf und der Mortalität. Nachdem erstmals wirksame Medikamente zur Therapie der IPF entwickelt wurden, bestätigt sich, dass in Studien, die einen positiven Effekt des Medikaments auf den Abfall der FVC zeigen konnten, auch ein zumindest numerisch positiver Effekt auf die Mortalität gefunden wurde, während in einer Studie, die keinen signifikanten Effekt auf den Abfall der FVC zeigte, auch die Mortalität nicht beeinflusst wurde. Insofern kann der Abfall der FVC als Surrogat für Mortalität bei IPF-Patienten in prospektiven interventionellen Studien als validiert betrachtet werden. Die FVC-Abnahme ist daher nicht nur ein anerkannter Studienendpunkt, sondern sollte auch für die Bewertung des patientenrelevanten Nutzens berücksichtigt werden.

\section{FVC Methodik}

$\nabla$

Die Spirometrie bezeichnet ein Messverfahren, welches die mobilisierbaren Lungenvolumina quantitativ erfasst. Das maximale ausatembare Volumen, gemessen aus maximaler Inspiration in einem forcierten Ausatemmanöver, bezeichnet man als forcierte Vitalkapazität (FVC). Die technischen Voraussetzungen und Durchführungsbestimmungen für diese Messung sind international standardisiert, sodass diese Methodik besonders

\section{Abstract \\ $\nabla$}

Spirometry is a highly standardized method which allows to measure the forced vital capacity (FVC) with high precision and reproducibility. In patients with IPF FVC is directly linked to the disease process which is characterized by scaring of alveoli and shrinkage of the lungs. Consequently, there is ample evidence form clinical studies that the decline of FVC over time is consistently associated with mortality in IPF. As for the first time effective drugs for the treatment of IPF are available it becomes obvious that in studies which could demonstrate that the drug reduces FVC decline, a numerical effect on mortality was also observed, while in one study where a significant effect on FVC decline was missed, there was also no change in mortality. Based on these studies FVC decline is a validated surrogate of mortality in IPF. It is concluded that FVC decline is not only accepted as an endpoint of clinical treatment trials in IPF but is also valid as a patient related outcome parameter which should be considered for the assessment of the efficacy of an IPF drug.

robuste und reproduzierbare Ergebnisse liefert [1]. Die Genauigkeit der Methode beträgt dabei $\pm 50 \mathrm{ml}$, bezogen auf ein maximales Messvolumen von 10 Litern; die Testvariabilität zwischen den Messungen beträgt maximal $\pm 3,5 \%$ oder $\pm 100 \mathrm{ml}$ [1]. Für die Beurteilung des Verlaufs von Lungenerkrankungen werden serielle Lungenfunktionsmessungen, speziell der FVC, zur Objektivierung des klinischen Eindrucks in der Pneumologie routinemäßig eingesetzt. 
Tab. 1 Analyse des Zusammenhangs zwischen der forcierten Vitalkapazität und der Gesamtmortalität [22]. ${ }^{1}$

\begin{tabular}{|c|c|c|c|c|c|c|}
\hline \multirow[t]{3}{*}{ Studie } & \multicolumn{3}{|c|}{ forcierte Vitalkapazität } & \multicolumn{3}{|c|}{ Gesamtmortalität } \\
\hline & \multicolumn{2}{|c|}{$\begin{array}{l}\text { Änderung vom Ausgangswert } \\
\text { (ml) }\end{array}$} & \multirow[t]{2}{*}{$\begin{array}{l}\text { Unterschied zwischen den } \\
\text { Therapiearmen }(95 \% \mathrm{Cl})\end{array}$} & \multicolumn{2}{|c|}{ Anzahl Todesfälle (\%) } & \multirow[t]{2}{*}{$\begin{array}{l}\text { Hazard Ratio für die Zeit } \\
\text { bis zum Tod }(95 \% \mathrm{Cl})\end{array}$} \\
\hline & $\begin{array}{l}\text { Studien- } \\
\text { medikament }\end{array}$ & Placebo & & $\begin{array}{l}\text { Studien- } \\
\text { medikament }\end{array}$ & Placebo & \\
\hline $\begin{array}{l}\text { Pirfenidone study } 2 \\
\text { (November 2008) }\end{array}$ & -318 & -475 & 157 (3 to 311$)$ & $14(8,0)$ & $20(11,5)$ & $0,65(0,33$ to 1,29$)$ \\
\hline $\begin{array}{l}\text { Pirfenidone study } 3 \\
\text { (November 2008) }\end{array}$ & -379 & -373 & $-6(-178$ to 167$)$ & $18(10,5)$ & $17(9,8)$ & $1,07(0,55$ to 2,08$)$ \\
\hline $\begin{array}{l}\text { Pirfenidone study } 1 \\
\text { (resubmitted; } \\
\text { February 2014) }\end{array}$ & -235 & -428 & 193 (96 to 289) & $12(4,3)$ & $21(7,6)$ & $0,57(0,28$ to 1,16$)$ \\
\hline $\begin{array}{l}\text { Nintedanib study } 1 \\
\text { (June 2010) }\end{array}$ & -60 & -191 & 131 (27 to 235$)$ & $7(8,1)$ & $9(10,3)$ & $0,73(0,27$ to 1,98$)$ \\
\hline $\begin{array}{l}\text { Nintedanib study } 2 \\
\text { (October 2013) }\end{array}$ & -115 & -240 & $125(78$ to 173$)$ & $13(4,2)$ & $13(6,4)$ & $0,63(0,29$ to 1,36$)$ \\
\hline $\begin{array}{l}\text { Nintedanib study } 3 \\
\text { (October 2013) }\end{array}$ & -114 & -207 & $94(45$ to 143$)$ & $22(6,7)$ & $20(9,1)$ & $0,74(0,40$ to 1,35$)$ \\
\hline
\end{tabular}

Legende: Die Studien wurden chronologisch aufgelistet. Die Studiennummer entspricht der im Label verwendeten Bezeichnung, zusätzlich ist der Monat angegeben, in dem die Rekrutierung abgeschlossen war.

Die für die FVC angegebenen Daten entsprechen dem absoluten Unterschied zwischen Ausgangswert und Woche 52 für die Pirfenidon-Studie 1, zwischen Ausgangswert und Woche 72 für die Pirfenidon-Studien 2 und 3 und zwischen Ausgangswert und Woche 52 für die Nintedanib-Studien. In den Pirfenidon-Studien wurde deskriptive Statistik verwendet, in den Nintedanib-Studien erfolgte eine Regressionsanalyse. Für die Gesamtmortalität wurde der Vitalstatus von der Randomisation bis zum Todeszeitpunkt analysiert, wobei alle Todesfälle berücksichtigt wurden, unabhängig von der Todesursache und auch unabhängig davon, ob der Patient noch das Medikament eingenommen hat oder nicht. Hazard ratios für die Zeit bis zum Tod basieren auf der Cox proportionalen-hazards Regressionsanalyse. Cl steht für „Confidenz Intervall“.

\section{FVC und idiopathische Lungenfibrose}

Die idiopathische Lungenfibrose (IPF) ist eine schwerwiegende, progredient verlaufende Erkrankung. Sie ist mit einer schlechten Prognose belastet [2]; das mediane Überleben ab Diagnose wird auf 2 - 4 Jahre geschätzt. Bei der IPF kommt es zu einer fortschreitenden Vernarbung der Lunge, gekennzeichnet durch die Vermehrung von Fibroblasten, die Ablagerung von extrazellulärer Matrix (u.a. Kollagenfasern), dem bindegewebigen Umbau der Lungenbläschen (Alveolen) und Zerstörung der Lungenarchitektur. Dies führt bei einem IPF-Patienten zu einer progredienten und irreversiblen Abnahme der Lungenfunktion, bis der Patient final im respiratorischen Versagen verstirbt. Dieser progrediente Fibrosierungsprozess führt zu einer Zerstörung der physiologischen Lungenarchitektur mit fortschreitender Vernarbung und Schrumpfung der Lunge, die sich unmittelbar in einer Abnahme der FVC manifestiert. Der individuelle Verlauf der Erkrankung kann dabei sehr unterschiedlich ausfallen [3]. Die fortschreitende Vernarbung und Schrumpfung der Lunge führt im Krankheitsverlauf der IPF fortschreitend und unumkehrbar zu einer Abnahme der FVC. Die jährliche FVC-Abnahme liegt bei IPF-Patienten im Durchschnitt bei ca. $150-250 \mathrm{ml}$, verglichen mit ca. $30-65 \mathrm{ml} /$ Jahr bei Gesunden [3 - 7]. Im Fall der IPF ist der Abfall der FVC daher unmittelbarer Ausdruck des Krankheitsgeschehens und spiegelt die Krankheitsprogression mit großer Präzision wider. Der Abfall der FVC ist daher bei der IPF ein Ausdruck der Erkrankung, welcher anderen unspezifischen klinischen Folgeerscheinungen der IPF (z.B. Dyspnoe, Leistungsminderung etc.) mindestens gleichwertig, unter Berücksichtigung der hohen Messgenauigkeit sogar überlegen ist.

Über die letzten zwei Dekaden wurden zahlreiche klinische Studien durchgeführt, um wirksame Behandlungsoptionen zu finden, die den Krankheitsverlauf positiv beeinflussen können [8]. Die Wahl geeigneter Studienendpunkte war in den vergangenen Jahren Gegenstand intensiver und kontroverser wissenschaftli- cher Diskussionen. Als primärer Studienendpunkt wäre der Nachweis einer Senkung der Gesamtmortalität sicherlich der unbestreitbarste Wirksamkeitsnachweis. Aufgrund der hohen erforderlichen Patientenzahlen, der nötigen Studiendauer und der sich daraus ergebenden ethischen Bedenken wird ein solcher Ansatz als nicht praktikabel angesehen [9-12]. Als sekundärer Endpunkt wird Mortalität in klinischen Studien zwar erfasst, jedoch sind diese Studien nicht ausreichend gepowert, um einen signifikanten Unterschied zeigen zu können. In dieser Situation bietet sich die Erfassung des Abfalls der FVC im Zeitverlauf als direktes Maß für Krankheitsprogression als Studienendpunkt an; dies umso mehr, als sich sowohl in retrospektiven als auch in prospektiven Studien eine klare Beziehung zwischen Abfall der FVC und Mortalität nachweisen ließ. Infolgedessen hat sich die serielle Messung der FVC zur Erfassung der Krankheitsprogression als primärer Endpunkt in klinischen Studien etabliert und ist auch weltweit von den Zulassungsbehörden als primärer Endpunkt in Studien akzeptiert.

\section{FVC und Mortalität}

Ein ganz grundsätzlicher Zusammenhang zwischen Lungenfunktion und Mortalität wurde in zahlreichen großen Studien belegt $[4,6,7,13-16]$. Speziell für Patienten mit IPF konnten mehrere Studien zeigen, dass eine Abnahme der FVC mit einer erhöhten Mortalität assoziiert ist [11,17 - 21]. In einer prospektiv erfassten Population von 1156 IPF-Patienten wurde das Mortalitätsrisiko von Patienten mit einer definierten FVC-Abnahme gegenüber stabilen Patienten ermittelt [21]. Patienten, die eine FVC-Abnahme von $>10 \%$ des Sollwerts innerhalb von 24 Wochen aufwiesen, hatten ein mehr als vierfach erhöhtes Mortalitätsrisiko, bezogen auf die folgenden 12 Monate, verglichen mit Patienten, deren FVC-Abnahme <5\% des Sollwerts in 24 Wochen betrug (HR 4,78; 95\% KI 3,12-7,33; p<0,001). Darüber hinaus wurde 
auch gezeigt, dass bereits eine geringere Abnahme von 5-10\% des Sollwerts mit einem erhöhten Mortalitätsrisiko assoziiert ist (HR 2,14; 95\% KI 1,43-3,20; p<0,001). Eine kategorische Abnahme der FVC von 5 oder 10\% des Sollwerts eignet sich daher, um ein erhöhtes Mortalitätsrisiko zu beschreiben. Dabei ist zu berücksichtigen, dass für die Beurteilung eines kategorischen FVC-Abfalls von $5-10 \%$ versus $>10 \%$ nicht allein der Unterschied in der Amplitude der Änderung relevant ist. Vielmehr ist, bedingt durch die intraindividuell bei seriellen FVC-Messungen zu erzielende Messgenauigkeit, die Irrtumswahrscheinlichkeit bei einem 5-10\%igen FVC Abfall ca. 10-20\%, während sie bei einem über $10 \%$ igen FVC-Abfall kleiner als 5\% ist. Dementsprechend ist in einer IPF-Population die Assoziation zwischen einem >10\%igen FVC-Abfall und dem Mortalitätsrisiko auch deshalb deutlich größer als für einen 5-10\%iger FVC-Abfall, weil die Zahl der fälschlich als progredient klassifizierten Patienten in letzterem Fall höher ist.

Für die Indikation IPF ist daher festzuhalten, dass der Nachweis einer Krankheitsprogression in Form eines Abfalls der FVC regelmäßig mit einem erhöhten Mortalitätsrisiko verbunden ist.

Allerdings war bislang nicht geklärt, ob eine Intervention, welche die FVC-Abnahme verlangsamt, auch einen positiven Effekt auf die Mortalität zeigen würde. In Abwesenheit wirksamer Medikamente war ein solcher Beweis auch nicht zu führen.

Seit 2011 sind Pirfenidon und seit 2015 Nintedanib in der Europäischen Union zur Behandlung der IPF zugelassen.

Die Mitarbeiter der FDA veröffentlichten kürzlich eine Analyse, bei der die FVC-Abnahmen und die Gesamtmortalität in den Pirfenidon- und Nintedanib-Studien untersucht wurden [22]. In Studien, bei denen Nintedanib oder Pirfenidon die FVC-Abnahme signifikant reduzierte, wurde immer auch ein numerischer Trend zugunsten einer verringerten Mortalität festgestellt (Hazard Ratio <1, vgl. Tab. 1). Bei der Beurteilung der Gesamtmortalität in den beiden Nintedanib-Studien in $\bullet$ Tab. 1 (Nintedanib study 2 und Nintedanib study 3) sind die \%-Angaben und Hazard Ratios relevant, während die Absolutzahlen wegen der 3:2-Randomisierung irreführend sind. In einer Studie, bei der Pirfenidon keinen signifikanten Effekt auf die FVC-Abnahme zeigte („Pirfenidone study 3“, vgl. Tab. 1), konnte entsprechend auch kein solcher Trend bezüglich der Mortalität gefunden werden.

Bislang konnten alle Studien, in denen Interventionen einen Effekt auf die FVC-Abnahme hatten, auch einen gleichgerichteten positiven Trend bezüglich der Mortalität zeigten. Damit ist eine zentrale Bedingung erfüllt, sodass die FVC als Surrogatparameter für Mortalität anerkannt werden kann.

\section{Fazit}

Die FVC-Abnahme hat sich als primärer Endpunkt in klinischen Studien bewährt. FVC-Messungen sind einfach durchzuführen und darüber hinaus sehr gut reproduzierbar. Es wurde gezeigt, dass die FVC-Abnahme Ausdruck der Krankheitsprogression und mit einer erhöhten Mortalität assoziiert ist. Darüber hinaus konnte in allen Studien, bei denen eine Intervention die FVC-Abnahme verlangsamte, auch ein gleichgerichteter positiver Trend auf die Abnahme der Mortalität festgestellt werden. Dies zeigt eindeutig, dass die FVC-Abnahme ein valider Studienendpunkt eigenständiger klinischer Relevanz und mit klarem Bezug zur Gesamtmortalität ist und als solcher bei der Bewertung des patientenrelevanten Nutzens einer Therapie berücksichtigt werden sollte.

\section{Interessenkonflikt}

$\nabla$

J. Behr hat Honorare für Vorträge und/oder Beratertätigkeit von Actelion, Allmiral, Bayer, Berlin-Chemie, Boehringer Ingelheim, Gilead, GSK, Intermune, Lilly, Novarties, Nycomed, Pfizer, Roche und Takeda erhalten. Er erhielt Forschungsförderung von Actelion, BMBF, Intermune, Ruhr-Universität Bochum und WATL.

R. Bonnet hat Honorare für Vorträge und/oder Beratertätigkeit von Intermune, Roche und Boehringer Ingelheim erhalten.

S. Gläser hat Vortragshonorare von Bayer, Boehringer Ingelheim und Roche erhalten sowie Studienteilnahme bei Boehringer Ingelheim, Actelion Pharma, Roche/Intermune, Gilead.

A. Günther hat Vortragshonorare von Intermune und Roche erhalten und ist Mitglied in Beratungsgremien von Intermune, Roche, Boehringer Ingelheim.

D. Koschel hat von Boehringer Ingelheim, Intermune und Roche Honorare für Vorträge und Beratertätigkeit sowie finanzielle Unterstützung für Kongressbesuche erhalten.

M. Kreuter hat Forschungsunterstützung von Roche/Intermune und Boehringer Ingelheim erhalten sowie Honorare für Referenten- und Beratungstätigkeiten von Roche/Intermune und Boehringer Ingelheim.

D. Kirsten hat Vortragshonorare von Boehringer Ingelheim und Intermune erhalten.

C. Krögel hat in den letzten 3 Jahren Vortragshonorare von ALK, Almiral, Astra-Zeneca, AWD Pharma, Berlin Chemie, Boehringer Ingelheim, Chiesi, CSL Behring, GlaxoSmithKline, Intermune, MEDA Pharma und Novartis erhalten.

P. Markart hat Honorare für Vortrags- und Beratertätigkeit sowie Reisekostenunterstützung für Kongressteilnahmen von Roche und Boehringer Ingelheim erhalten.

C. Neurohr hat von den Firmen Roche und Boehringer Ingelheim finanzielle Unterstützung im Rahmen von Kongressbesuchen und Advisory Boards erhalten.

A. Prasse hat Honorare für Vortrags- und/oder Beratertätigkeit von Boehringer Ingelheim und Intermune/Roche sowie Forschungsunterstützung von Biogen Idec erhalten.

J. Schreiber hat Beratungs- und Vortragshonorare von Boehringer Ingelheim und Roche (ehemals Intermune) erhalten. Er war in Beratergremien von Berlin-Chemie, Chiesi, Intermune, Boehringer Ingelheim sowie Novartis tätig und erhielt eine Forschungsunterstützung von Novartis Bencard, Boehringer Ingelheim und Roche.

U. Costabel hat Honorare für Vorträge und/oder Beratertätigkeit von Bayer, Boehringer Ingelheim, Centocor, Gilead, Intermune, Roche und Forschungsförderung von Boehringer Ingelheim und Intermune erhalten.

F. Bonella, C. Grohé, J. Müller-Quernheim, M. Pfeifer, N. Schönfeld, $\mathrm{H}$. Wirtz und C. Witt geben an, dass kein Interessenkonflikt besteht.

\section{Institute}

${ }^{1}$ Medizinische Klinik und Poliklinik V, Klinikum der Universität München und Asklepios Fachkliniken München-Gauting, Comprehensive Pneumology Center, Mitglied des Deutschen Zentrums für Lungenforschung

${ }^{2}$ Ruhrlandklinik, Westdeutsches Lungenzentrum am Universitätsklinikum Essen $\mathrm{gGmbH}$

Abteilung Pneumologie, Zentralklinik Bad Berka GmbH

${ }^{4}$ Klinik für Innere Medizin B - Bereich Pneumologie, Infektiologie und

Weaningzentrum Forschungsbereich Pneumologie und Pneumologische Epidemiologie Universitätsmedizin Greifswald

${ }^{5}$ Pneumologische Klinik Evangelische Lungenklinik Berlin - Krankenhausbetriebs $\mathrm{gGmbH}$ 
${ }^{6}$ Medizinische Klinik III, Pneumologie und Internistische Intensivmedizin Agaplesion Evangelisches Krankenhaus Mittelhessen und Schwerpunkt „Fibrosierende Lungenerkrankungen“, Universitätsklinikum Gießen Marburg $\mathrm{GmbH}$, Mitglied des Deutschen Zentrums für Lungenforschung

${ }^{7}$ Abteilung Innere Medizin/Pneumologie, Fachkrankenhaus Coswig $\mathrm{GmbH}$ Zentrum für Pneumologie, Allergologie, Beatmungsmedizin, Thorax- und Gefäßchirurgie

${ }^{8}$ Pneumologie und Beatmungsmedizin, Zentrum für seltene und interstitielle Lungenerkrankungen Thoraxklinik, Universitätsklinikum Heidelberg, Mitglied des Deutschen Zentrums für Lungenforschung

${ }^{9}$ Lungenclinic Grosshansdorf

${ }^{10}$ Abteilung Pneumologie \& Allergologie/Immunologie, Medizinische Klinik I, Klinikum der Universität Jena

${ }^{11}$ Medizinische Klinik und Poliklinik II, Universitätsklinikum Gießen und Marburg GmbH, Standort Gießen und Medizinische Klinik V (Pneumologie), Herz-Thorax-Zentrum, Klinikum Fulda gAG

${ }^{12}$ Abteilung für Pneumologie, Universitätsklinikum Freiburg

${ }^{13}$ Medizinische Klinik und Poliklinik V, Klinikum der Universität München, Comprehensive Pneumology Center, Mitglied des Deutschen Zentrums für Lungenforschung

${ }^{14}$ Klinik Donaustauf, Klinik für Pneumologie und konservative Intensivmedizin Krankenhaus Barmherzige Brüder, Regensburg, Klinik und Poliklinik für Innere Medizin II Universität Regensburg

${ }^{15}$ Abteilung Pneumologie, Medizinische Hochschule Hannover

${ }^{16}$ Klinik für Pneumologie, Lungenklinik Heckeshorn, HELIOS Klinikum Emil von Behring, Berlin

${ }^{17}$ Klinik für Pneumologie, Universitätsklinikum der Otto-von-GuerickeUniversität, Magdeburg

${ }_{18}$ Abteilung Pneumologie, Universitätsklinikum Leipzig

${ }^{19}$ Abteilung Pneumologie und Transplantation, Universitätsklinikum Charité, Berlin

${ }^{20}$ Senior Consultant, Interstitielle und Seltene Lungenkrankheiten, Ruhrlandklinik, Westdeutsches Lungenzentrum am Universitätsklinikum Essen $\mathrm{gGmbH}$

\section{Literatur}

1 Miller MR, Hankinson J, Brusasco Vet al. Standardisation of spirometry. Eur Respir J 2005; 26: 319 - 338

2 Behr J, Günther A, Ammenwerth W et al. S2K-Leitlinie zur Diagnostik und Therapie der idiopathischen Lungenfibrose. Pneumologie 2013; 67: $81-111$

3 Ley B, Collard HR, King TE Jr. Clinical course and prediction of survival in idiopathic pulmonary fibrosis. Am J Respir Crit Care Med 2011; 183: $431-440$

4 Griffith KA, Sherrill DL, Siegel EM et al. Predictors of loss of lung function in the elderly: the Cardiovascular Health Study. Am J Respir Crit Care Med 2001; 163: $61-68$

5 Alexeeff SE, Litonjua AA, Sparrow $D$ et al. Statin use reduces decline in lung function: VA Normative Aging Study. Am J Respir Crit Care Med 2007; 176: $742-747$

6 Braendli 0 , Schindler $C$, Kunzli $N$ et al. Lung function in healthy never smoking adults: reference values and lower limits of normal of a Swiss population. Thorax 1996; 51: $277-283$
7 Janssens JP. Aging of the respiratory system: impact on pulmonary function tests and adaptation to exertion. Clin Chest Med 2005; 26: 469 - 484 vi-vii.

8 Antoniou KA, Wells U. Acute Exacerbations of Idiopathic Pulmonary Fibrosis Respiration. 2013; 86: 265 - 274

9 Raghu G, Collard HR, Anstrom KJ et al. Idiopathic Pulmonary Fibrosis: Clinically Meaningful Primary Endpoints in Phase 3 Clinical Trials. American Journal of Respiratory and Critical Care Medicine 2012; 185: $1044-1048$

10 Wells AU, Behr J, Costabel $U$ et al. Triple therapy in idiopathic pulmonary fibrosis: an alarming press release. Eur Respir J 2012; 39: 805 806

11 Collard HR, Brown KK, Martinez FJ et al. Study design implications of death and hospitalization as endpoints in idiopathic pulmonary fibrosis. Chest 2014; 146: $1256-1262$

12 King TE Jr, Albera C, Bradford WZ et al. All-cause mortality rate in patients with idiopathic pulmonary fibrosis. Implications for the design and execution of clinical trials. Am J Respir Crit Care Med 2014; 189: $825-833$

13 Batty GD, Gunnell D, Langenberg C et al. Adult height and lung function as markers of life course exposures: associations with risk factors and cause-specific mortality. Eur J Epidemiol 2006; 21: 795-801

14 Buchman A, Boyle $P$, Wilson $R$ et al. Pulmonary function, muscle strength and mortality in old age. Mech Ageing Dev 2008; 129: 625 631

15 Sabia S, Shipley M, Elbaz A et al. Why does lung function predict mortality? Results from the Whitehall II cohort study Am J Epidemiol 2010; 172: 1415 - 1423

$16 \operatorname{Sin} \mathrm{DD}, \mathrm{Wu} \mathrm{L}, \mathrm{Man} \mathrm{SF}$. The relationship between reduced lung function and cardiovascular mortality: a population-based study and a systematic review of the literature. Chest 2005; 127: 1952 - 1959

17 Flaherty KR, Mumford JA, Murray S et al. Prognostic implications of physiologic and radiographic changes in idiopathic interstitial pneumonia. Am J Respir Crit Care Med 2003; 168: 543- 548

18 Jegal Y, Kim DS, Shim TS et al. Physiology is a stronger predictor of survival than pathology in fibrotic interstitial pneumonia. Am J Respir Crit Care Med 2005; 171: 639-644

19 Latsi PI, du Bois RM, Nicholson AG et al. Fibrotic idiopathic interstitial pneumonia: the prognostic value of longitudinal functional trends. Am J Respir Crit Care Med 2003; 168: 531 - 537

20 Zappala CJ, Latsi PI, Nicholson AG et al. Marginal decline in forced vital capacity is associated with a poor outcome in idiopathic pulmonary fibrosis. Eur Respir J 2010; 35: $830-836$

$21 \mathrm{du}$ Bois RM, Weycker D, Albera $C$ et al. Forced vital capacity in patients with idiopathic pulmonary fibrosis: test properties and minimal clinically important difference. Am J Respir Crit Care Med 2011; 184: $1382-1389$

22 Karimi-Shah BA, Chowdhury BA. Forced Vital Capacity in Idiopathic Pulmonary Fibrosis - FDA Review of Pirfenidone and Nintedanib. N Engl J Med 2015; 372: 1189-1191 